Rev. Inst. Flor. v. 29 n. 2 p. $145-155$ dez. 2017

http://dx.doi.org/10.24278/2178-5031.201729201

ISSN impresso 0103-2674/on-line 2178-5031

\title{
ASPECTOS FISIOLÓGICOS DO EUCALIPTO EM CONVIVÊNCIA COM TRÊS ESPÉCIES DE PLANTAS DANINHAS ${ }^{1}$
}

\section{PHYSIOLOGICAL ASPECTS OF EUCALYPTUS IN COEXISTENCE WITH THREE WEED SPECIES}

\author{
Laís Araújo FAUSTINO²; Guilherme Pereira QUEIROZ²; Gustavo Antônio Mendes PEREIRA²,4; \\ Evander Alves FERREIRA'; ${ }^{3}$ Lino Roberto FERREIRA ${ }^{2}$
}

\begin{abstract}
RESUMO - As espécies daninhas Commelinea bengalensis (trapoeraba), Ipomoeia grandifolia (corda-de-viola) e Urochloa brizantha (braquiária) são comumente encontradas em competição com a cultura do eucalipto, sendo de difícil manejo com a utilização única de um mesmo herbicida. A avaliação da agressividade dessas espécies, por meio de variáveis fisiológicas medidas na cultura, pode ser útil no estabelecimento de estratégias de controle alternativas à aplicação de herbicidas. Deste modo, este trabalho foi proposto com o objetivo de avaliar o efeito do período de convivência da trapoeraba, da corda-de-viola e da braquiária nas características fisiológicas de plantas de eucalipto. $\mathrm{O}$ delineamento experimental utilizado foi em blocos casualizados (DBC) com quatro repetições, no esquema fatorial $3 \times 3$, sendo o fator A representado pelas três espécies avaliadas: C. bengalensis, I. grandifolia e U. brizantha, e o fator B representando os três períodos de convivência da cultura com as plantas daninhas: testemunha (cultivadas na ausência de plantas daninhas), 60 dias de convivência e 105 dias de convivência do eucalipto com as espécies daninhas. As três espécies avaliadas promoveram efeitos negativos nas características fisiológicas das plantas de eucalipto causando redução na taxa fotossintética e na eficiência do uso da água, além de incremento na porcentagem, na concentração do carbono interno e na taxa transpiratória. As três espécies avaliadas afetaram de maneira similar as características fisiológicas da cultura.
\end{abstract}

Palavras-chave: Eucalyptus spp.; Commelinea bengalensis; Ipomoeia grandifolia; Urochloa brizantha; potencial competitivo.

\begin{abstract}
The weeds Commelinea bengalensis (trapoeraba), Ipomoea grandifolia (corda-de-viola) and Urochloa brizantha (braquiária) are commonly found in competition with eucalyptus cultivation, and unwieldy for the sole use of herbicides. The aggressiveness evaluation of these species by physiological variables measured in culture may be useful in the establishment of alternative control strategies to herbicide application. Thus, this study aimed to evaluate the effect of coexistence period of trapoeraba, corda-de-viola and braquiária on physiological characteristics of eucalyptus plants.
\end{abstract}

\footnotetext{
${ }^{1}$ Recebido para análise em 17.02.2017. Aceito para publicação em 05.09.2017.

²Universidade Federal de Viçosa, Campus de Viçosa, Avenida Peter Henry Rolfs, s/n, Campus Universitário, 36570-900, Viçosa, MG, Brasil.

${ }^{3}$ Universidade Federal dos Vales do Jequitinhonha e Mucuri, Campus JK, Rodovia MGT 367 - Km 583, n 5000, Alto da Jacuba, 39100-000, Diamantina, MG, Brasil.

${ }^{4}$ Autor para correspondência: Gustavo Antônio Mendes Pereira - gustavogamp@hotmail.com
} 
The experimental design was a randomized block design - RBD with four replications, in a factorial $3 \times 3$, being the factor represented by three species assessed: $C$. bengalensis, I. grandifolia and $U$. brizantha and factor B representing the three periods of coexistence culture with weeds: control (cultured without weeds), 60 days living and 105 two eucalyptus coexistence with weeds. The three species evaluated promoted negative effects on eucalyptus physiological characteristics promoting reduction in photosynthetic rate and water use efficiency, and percentage increase in the concentration of internal carbon and transpiration rate. The three evaluated species affected in a similar way the physiological characteristics of culture.

Keywords: Eucalyptus spp.; Commelinea bengalensis; Ipomoeia grandifolia; Urochloa brizantha; competitive potential.

\section{INTRODUÇÃO}

Um dos grandes problemas enfrentados pelos produtores nas plantações de eucalipto é a infestação de plantas daninhas, cujo manejo assume papel de destaque entre os tratos culturais, apresentando reflexos diretos na produtividade e nos custos de produção (Machado et al., 2010). Para que não ocorram perdas de produtividade, torna-se necessária a redução dos fatores limitantes ao crescimento e desenvolvimento das espécies de eucalipto, sendo que a interferência das plantas daninhas é considerada um dos maiores problemas na implantação, na manutenção e na reforma de plantios de eucalipto, pois pode comprometer a produtividade dos plantios, além de aumentar os riscos de incêndio e reduzir o rendimento da colheita (Ferreira et al., 2016).

O potencial competitivo das plantas pelos recursos do meio varia em função da espécie presente na área (Rigoli et al., 2008), do nível populacional (Vidal et al., 2004), da época de emergência em relação à cultura (Silva et al., 2007), do período de convivência da cultura com as plantas daninhas (Faustino, 2015) e das características competitivas dos cultivares (Galon et al., 2007).

A competição das plantas daninhas com as culturas leva ao menor fornecimento de alguns recursos para as plantas, ocasionando deficiências que culminam em alterações nas características fisiológicas relacionadas com a fotossíntese, como a deficiência hídrica (Floss, 2011), nutricional (Melo et al., 2006) e a baixa qualidade ou quantidade de luminosidade (Sharkey e Raschke, 1981). Essas limitações podem levar a alterações na condutância estomática, na concentração interna de gases e, consequentemente, na atividade fotossintética e uso eficiente da água. $\mathrm{O}$ uso mais eficiente da água está diretamente relacionado ao tempo de abertura estomática, pois, enquanto o dióxido de carbono $\left(\mathrm{CO}_{2}\right)$ penetra na folha, a água é perdida pela transpiração, com intensidade variável, o que é dependente do gradiente de potencial entre a superfície foliar e a atmosfera (Concenço et al., 2007, 2009).

As plantas daninhas Commelinea bengalensis, Ipomoeia grandifolia e Urochloa brizantha são comumente encontradas em competição com a cultura do eucalipto, sendo de difícil manejo com a utilização única de herbicidas (Winkler et al., 2002; Vidal et al., 2004; Faustino, 2015). A hipótese deste trabalho é que a avaliação da agressividade dessas espécies, por meio de variáveis fisiológicas medidas na cultura, pode ser útil no estabelecimento de estratégias de controle alternativas à aplicação de herbicidas.

Deste modo, este trabalho foi proposto com o objetivo de avaliar o efeito do período de convivência da trapoeraba, da corda-de-viola e da braquiária nas características fisiológicas de plantas de eucalipto. 
FAUSTINO, L.A. et al. Eucalipto em convivência com plantas daninhas.

\section{MATERIAL E MÉTODOS}

O experimento foi conduzido no período de 18 de fevereiro a 03 de junho de 2014, na Estação Experimental Diogo Alves de Melo, Campus da Universidade Federal de Viçosa, pertencente ao Departamento de Fitotecnia.

O delineamento experimental utilizado foi em blocos casualizados (DBC) com quatro repetições, no esquema fatorial $3 \times 3$, sendo o fator A representado pelas três espécies avaliadas: C. bengalensis, I. grandifolia e U. brizantha e o fator $\mathrm{B}$ representando os três períodos de convivência da cultura com as plantas daninhas: testemunha (cultivadas na ausência de plantas daninhas), 60 dias de convivência e 105 dias de convivência do eucalipto com as espécies daninhas.
As unidades experimentais foram constituídas por vasos de $110 \mathrm{dm}^{3}$ de solo em campo, com uma planta de eucalipto e três da espécie daninha, e irrigadas de modo que mantivessem adequada disponibilidade de água, acompanhando as variações climáticas durante a época de condução (Figura 1).

Para enchimento dos vasos utilizou-se um solo de textura muito argilosa com as características químicas e físicas apresentadas na Tabela 1.

Para adequação do substrato quanto à nutrição foram aplicados $o$ equivalente $a$ $1,5 \mathrm{t} \mathrm{ha}^{-1}$ de calcário dolomítico e 10,0;0,45 e $0,38 \mathrm{~kg} \mathrm{~m}^{-3}$ de superfosfato simples, sulfato de amônio e cloreto de potássio, respectivamente.

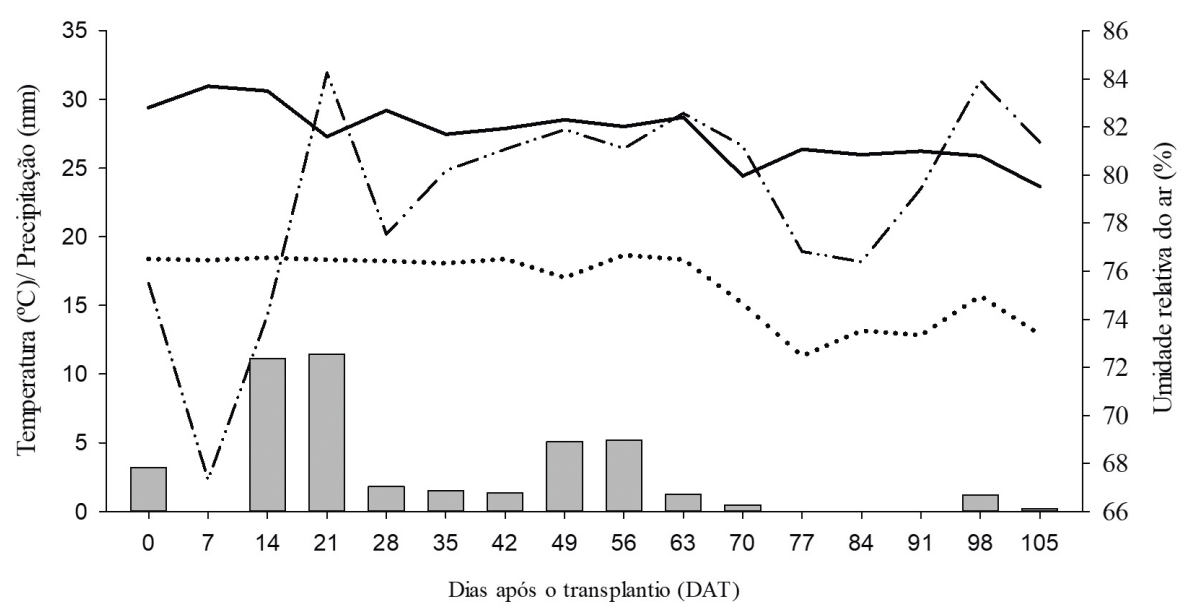

Figura 1. Médias semanais de temperatura máxima $\left({ }^{\circ} \mathrm{C}\right)$, temperatura mínima $\left({ }^{\circ} \mathrm{C}\right.$ ), precipitação (milímetros) e umidade relativa do ar (\%) observadas durante a condução do experimento.

Figure 1. Weekly averages of maximum temperature $\left({ }^{\circ} \mathrm{C}\right)$, minimum temperature $\left({ }^{\circ} \mathrm{C}\right)$, precipitation (millimeters) and relative humidity (\%) observed during the conduction of the experiment.

Tabela 1. Características químicas e físicas do solo usado para enchimento dos vasos.

Table 1. Chemical and physical soil characteristics used to fill pots.

\begin{tabular}{|c|c|c|c|c|c|c|c|c|c|c|}
\hline $\mathrm{pH}$ & $\mathrm{P}$ & $\mathrm{K}$ & $\mathrm{Ca}^{2}+$ & $\mathrm{Mg}^{2+}$ & $\mathrm{Al}^{3}+$ & $\mathrm{H}+\mathrm{Al}$ & $(\mathrm{t})$ & $\mathrm{V}$ & $\mathrm{m}$ & $\mathrm{MO}$ \\
\hline $\mathrm{H}_{2} \mathrm{O}$ & \multicolumn{2}{|c|}{$-m g d^{-3}--$} & \multicolumn{5}{|c|}{--------------cmolc dm ${ }^{-3}$------------ } & \multicolumn{3}{|c|}{$---\%$} \\
\hline 5,10 & 0,60 & 9,00 & 0,04 & 0,06 & 1,60 & 5,30 & 1,66 & 2,90 & 90,70 & 2,07 \\
\hline \multicolumn{3}{|c|}{ A. Grossa } & A. Fina & \multicolumn{2}{|c|}{ Silte } & \multicolumn{2}{|l|}{ Argila } & \multirow{2}{*}{\multicolumn{3}{|c|}{ Classe Textural }} \\
\hline \multicolumn{8}{|c|}{ - } & & & \\
\hline & 00 & &, 00 & 17, & & 62,00 & & \multicolumn{3}{|c|}{ Muito Argiloso } \\
\hline
\end{tabular}


FAUSTINO, L.A. et al. Eucalipto em convivência com plantas daninhas.

Mudas padronizadas do clone AEC144, híbrido Eucalyptus urophylla $\mathrm{x}$ Eucalyptus grandis, com média de $23 \mathrm{~cm}$ de altura e três meses de idade, foram transplantadas no centro do vaso simultaneamente ao transplantio de três plantas de C. benghalensis, ou I. grandifolia, ou $U$. brizantha com 4 a 6 folhas.

A adubação com micronutrientes nas doses de $10 ; 17,5$ e $100 \mathrm{mg}$ vaso $^{-1}$, respectivamente de boro, cobre e zinco, foi realizada por via líquida e parcelada em duas vezes, aos 10 e 30 dias após o transplantio (DAT) das mudas de eucalipto.

As avaliações fisiológicas foram realizadas aos 105 DAT em plantas que conviveram com as daninhas por diferentes períodos: sem convivência durante todo o tempo; 60 dias de convivência e 105 dias de convivência.

As avaliações foram realizadas no terço médio da folha adulta mais jovem das plantas de eucalipto, o que corresponde à quarta ou quinta folha completamente expandida contando do meristema apical para a base. Foi utilizado um analisador de gases no infravermelho (IRGA), marca ADC, modelo LCA 4 (Analytical Development Co. Ltd, Hoddesdon, UK), em casa de vegetação aberta, permitindo livre circulação do ar. Cada bloco foi avaliado em um dia, entre 8 e 10 horas da manhã, de forma que mantivesse as condições ambientais homogêneas durante a avaliação.
As variáveis avaliadas foram consumo de $\mathrm{CO}_{2}\left(\Delta \mathrm{C}-\mu \mathrm{mol} \mathrm{mol}^{-1}\right)$, carbono interno $\left(\mathrm{Ci}-\mu \mathrm{mol} \mathrm{mol}^{-1}\right)$, condutância estomática de vapores de água (Gs $-\mathrm{mol} \mathrm{m}^{-1} \mathrm{~s}^{-1}$ ), pressão de vapor na câmara subestomática e taxa de transpiração $\left(\mathrm{E}-\mathrm{mol} \mathrm{H}_{2} \mathrm{O} \mathrm{m}^{-2} \mathrm{~s}^{-1}\right)$, taxa fotossintética (A $-\mu \mathrm{mol} \mathrm{m} \mathrm{m}^{-2} \mathrm{~s}^{-1}$ ), sendo calculada ainda a eficiência do uso da água ( $\left.\mathrm{UE}-\mathrm{mol} \mathrm{CO}_{2} \mathrm{~mol} \mathrm{H}_{2} \mathrm{O}^{-1}\right)$ pela relação entre quantidade de $\mathrm{CO}_{2}$ fixado pela fotossíntese, temperatura foliar e ambiente $\mathrm{e}$ quantidade de água transpirada.

Os dados obtidos foram transformados em porcentagem em relação à testemunha e submetidos à análise de variância e ao Teste de Tukey a $5 \%$ de probabilidade de erro.

\section{RESULTADOS E DISCUSSÃO}

As três espécies daninhas avaliadas não mostraram diferença na porcentagem da taxa fotossintética em relação à testemunha $(\mathrm{A} \%)$ aos 60 e 105 dias de convivência com o eucalipto. Ao se avaliar o período de convivência dentro de cada espécie, verificou-se diferença da $\mathrm{A} \%$ entre a testemunha e os períodos de convivência, destacando-se que o período de convivência de 60 e 105 dias afetou negativamente essa variável, sendo que o decréscimo na taxa fotossintética foi de aproximadamente 60, 54 e 55\% nas espécies trapoeraba, corda-de-viola e braquiária, respectivamente, no maior período de convivência da cultura com as plantas daninhas (Tabela 2).

Tabela 2. Porcentagem da taxa fotossintética em relação à testemunha ( $\mathrm{A} \%)$ de plantas de eucalipto em diferentes períodos de convivência com plantas daninhas.

Table 2. Percentage of the photosynthetic rate in relation to the control $(\mathrm{A} \%)$ of eucalyptus plants in different periods of cohabitation with weeds.

Períodos de convivência Espécies

\begin{tabular}{ccc}
\multicolumn{3}{c}{ Espécies } \\
\hline Commelinea bengalensis & Ipomoeia grandifolia & Urochloa brizantha \\
\hline $100,00 \mathrm{~A}^{*}$ & $100,00 \mathrm{~A}$ & $100,00 \mathrm{~A}$ \\
62,53 a B & 61,11 a B & 59,62 a B \\
41,62 a B & 55,34 a B & 46,24 a B
\end{tabular}

\begin{tabular}{lccc}
\hline Testemunha & $100,00 \mathrm{~A}^{*}$ & $100,00 \mathrm{~A}$ & $100,00 \mathrm{~A}$ \\
60 dias & $62,53 \mathrm{a} \mathrm{B}$ & 61,11 a B & 59,62 a B \\
105 dias & $41,62 \mathrm{a} \mathrm{B}$ & 55,34 a B & 46,24 a B \\
\hline $\mathrm{CV}(\%)$ & & 19,16 & \\
\hline
\end{tabular}

*Médias seguidas pela mesma letra minúscula na linha e maiúscula na coluna não diferem entre si pelo Teste de Tukey a 5\% de probabilidade de erro.

*Averages followed by the same lowercase letter in the row and uppercase in the column do not differ from each other by the Tukey test at $5 \%$ probability of error. 
FAUSTINO, L.A. et al. Eucalipto em convivência com plantas daninhas.

Ferreira et al. (2016) observaram que a convivência por 48 dias após o transplantio das mudas de eucalipto com espécies do gênero Urochloa afetaram negativamente a taxa fotossintética das plantas de eucalipto. A taxa fotossintética pode sofrer interferência de diversos fatores, tais como a redução da disponibilidade de recursos proporcionados pela competição com plantas daninhas, assim uma espécie pode privar a outra de recursos como a luz, água e nutrientes afetando as características fisiológicas de ambas as espécies ou plantas da mesma espécie em estado de competição (Concenço et al., 2007).

De acordo com Silva et al. (2001), E. citriodora e E. grandis apresentaram o mesmo comportamento quando submetidas à competição com diferentes densidades de $U$. brizantha por vaso e diferentes níveis de água no solo por 70 dias. A taxa fotossintética apresentou menor valor, independente dos níveis de água e da densidade de $U$. brizantha, mostrando a interferência negativa desta planta daninha com o eucalipto.

A competição entre plantas daninhas e culturas é um fator crítico para o desenvolvimento da cultura quando a espécie daninha se estabelece junto ou primeiro que a cultura
(Radosevich et al., 1996). Todavia, se a cultura se estabelecer primeiro, em função da espécie cultivada, do seu vigor, da velocidade de crescimento inicial e da densidade de plantio, ela poderá cobrir rapidamente o solo, podendo excluir ou inibir significativamente o crescimento das plantas daninhas. No presente trabalho, o transplantio das mudas das plantas daninhas foi realizado no momento do transplantio das mudas do eucalipto.

Foram constatadas diferenças na condutância estomática (GS\%), tanto quando as plantas de eucalipto conviveram com espécies daninhas diferentes como quando em convivência por 60 quanto por 150 dias. Plantas de trapoeraba mostraram maior condutância estomática quando comparadas com as demais espécies, destacando-se que, quando competindo com braquiária, as plantas de eucalipto mostraram redução de aproximadamente $37 \%$ na Gs\% no maior período de convivência. Plantas de eucalipto mostraram incremento nos valores de Gs\% em relação à testemunha quando submetidas a $60 \mathrm{e}$ 150 dias de convivência com a trapoeraba e a corda-de-viola, no entanto, ao competir com braquiária, o eucalipto mostrou decréscimos nos valores de Gs\% em relação a testemunha (Tabela 3).

Tabela 3. Porcentagem da condutância estomática relação à testemunha (Gs\%) de plantas de eucalipto em diferentes períodos de convivência com plantas daninhas.

Table 3. Percentage of stomatal conductance relative to the control (Gs\%) of eucalyptus plants in different periods of cohabitation with weeds.

Períodos de convivência

Espécies

Commelinea bengalensis Ipomoeia grandifolia

Urochloa brizantha

\begin{tabular}{lccc}
\hline Testemunha & $100,00 \mathrm{C}^{*}$ & $100,00 \mathrm{C}$ & $100,00 \mathrm{~A}$ \\
60 dias & $183,83 \mathrm{a} \mathrm{D}$ & $131,08 \mathrm{~b} \mathrm{D}$ & $68,27 \mathrm{c} \mathrm{B}$ \\
105 dias & $229,044 \mathrm{a} \mathrm{A}$ & $190,39 \mathrm{~b} \mathrm{~A}$ & $63,47 \mathrm{c} \mathrm{B}$ \\
\hline $\mathrm{CV}(\%)$ & & 30,62 & \\
\hline
\end{tabular}

*Médias seguidas pela mesma letra minúscula na linha e maiúscula na coluna não diferem entre si pelo Teste de Tukey a 5\% de probabilidade de erro.

*Averages followed by the same lowercase letter in the row and uppercase in the column do not differ from each other by the Tukey test at $5 \%$ probability of error. 
A condutância estomática é o mecanismo fisiológico que as plantas terrestres vasculares possuem para o controle da transpiração (Messinger et al., 2006) e fornecimento de $\mathrm{CO}_{2}$. As principais funções adaptativas dos estômatos são a otimização das trocas gasosas foliares, redução da desidratação, especialmente em folhas com potencial hídrico abaixo do ponto de cavitação do xilema (Tyree e Sperry, 1988), e regulação da temperatura foliar por meio do resfriamento transpiracional (Jones, 1998).

Aos 60 dias de convivência do eucalipto com as plantas daninhas, não foi verificada diferença na porcentagem de carbono interno em relação à testemunha $(\mathrm{Ci} \%)$ em planta de eucalipto. Aos 105 dias de convivência com as plantas daninhas, o eucalipto mostrou maiores valores de $\mathrm{Ci} \%$ quando em competição com a braquiária. Ao se avaliar o efeito do período de convivência dentro de cada espécie, contatou-se que a $\mathrm{Ci} \%$ apresentou acréscimo em todas as espécies avaliadas em relação à testemunha aos 60 e 105 dias de convivência da cultura com as espécies daninhas (Tabela 4).

A Ci é considerada variável fisiológica influenciada por fatores ambientais, como disponibilidade hídrica, de luz e energia, entre outros. $\mathrm{O}$ incremento na $\mathrm{Ci}$ pode indicar uma tentativa da planta de escapar do estresse gerado pela competição pelos recursos do ambiente (Matos et al., 2013).
Plantas de eucalipto mostraram maiores valores de porcentagem da taxa transpiratória em relação à testemunha (E\%) quando competindo com trapoeraba no período de 60 dias de convivência, já aos 105 dias, não foi observada diferença da $\mathrm{E} \%$ para o eucalipto competindo com as três espécies avaliadas. $\mathrm{O}$ eucalipto quando cultivado com as três espécies mostrou maiores valores de E\% aos 60 e 105 dias de convivência, diferindo da testemunha cultivada na ausência de competição (Tabela 5).

Não foi observada diferença na porcentagem da temperatura foliar em relação à testemunha $(\mathrm{TF} \%)$ de plantas de eucalipto convivendo com as três espécies daninhas e entre os períodos de convivência das plantas daninhas com a cultura (Tabela 6). O metabolismo do vegetal causa incremento na temperatura da folha, assim, via de regra, a temperatura da folha é superior à do ar ao seu redor (Concenço et al., 2007). Assim, incrementos no metabolismo, em situações de estresse como interferência de plantas daninhas, podem ser indiretamente aferidos em função do gradiente entre temperatura da folha e do ar, destacando-se que, no presente trabalho, os tratamentos não interferiram na temperatura da folha de nenhuma espécie avaliada.

Tabela 4. Porcentagem da concentração do carbono interno em relação à testemunha (Ci\%) de plantas de eucalipto em diferentes períodos de convivência com plantas daninhas.

Table 4. Percentage of the internal carbon concentration in relation to the control (Ci\%) of eucalyptus plants in different periods of weed coexistence.

\begin{tabular}{lccc}
\hline Períodos de convivência & \multicolumn{3}{c}{ Espécies } \\
\cline { 2 - 4 } & Commelinea bengalensis & Ipomoeia grandifolia & Urochloa brizantha \\
\hline Testemunha & $100,00 \mathrm{~B}^{*}$ & $100,00 \mathrm{~B}$ & $100,00 \mathrm{~B}$ \\
60 dias & $130,38 \mathrm{a} \mathrm{A}$ & $128,35 \mathrm{a} \mathrm{A}$ & $138,56 \mathrm{a} \mathrm{A}$ \\
105 dias & $131,22 \mathrm{~b} \mathrm{~A}$ & $133,57 \mathrm{~b} \mathrm{~A}$ & 145,97 a A \\
\hline
\end{tabular}

$\mathrm{CV}(\%) \quad 5,37$

*Médias seguidas pela mesma letra minúscula na linha e maiúscula na coluna não diferem entre si pelo Teste de Tukey a $5 \%$ de probabilidade de erro.

*Averages followed by the same lowercase letter in the row and uppercase in the column do not differ from each other by the Tukey test at $5 \%$ probability of error. 
FAUSTINO, L.A. et al. Eucalipto em convivência com plantas daninhas.

Tabela 5. Porcentagem da taxa transpiratória em relação à testemunha $(\mathrm{E} \%)$ de plantas de eucalipto em diferentes períodos de convivência com plantas daninhas.

Table 5. Percentage of the transpiratory rate in relation to the control (E\%) of eucalyptus plants in different periods of coexistence with weeds.

\begin{tabular}{|c|c|c|c|}
\hline \multirow[t]{2}{*}{ Períodos de convivência } & \multicolumn{3}{|c|}{ Espécies } \\
\hline & Commelinea bengalensis & Ipomoeia grandifolia & Urochloa brizantha \\
\hline Testemunha & $100,00 \mathrm{~B}^{*}$ & $100,00 \mathrm{~B}$ & $100,00 \mathrm{~B}$ \\
\hline 60 dias & 175,03 a $\mathrm{A}$ & $121,08 \mathrm{~b} \mathrm{~A}$ & 134,93 b A \\
\hline 105 dias & 149,25 a A & 133,40 a $\mathrm{A}$ & 120,25 a A \\
\hline $\mathrm{CV}(\%)$ & & 15,97 & \\
\hline
\end{tabular}

*Médias seguidas pela mesma letra minúscula na linha e maiúscula na coluna não diferem entre si pelo Teste de Tukey a $5 \%$ de probabilidade de erro.

*Averages followed by the same lowercase letter in the row and uppercase in the column do not differ from each other by the Tukey test at $5 \%$ probability of error.

Tabela 6. Porcentagem da temperatura foliar em relação à testemunha $(\mathrm{TF} \%)$ de plantas de eucalipto em diferentes períodos de convivência com plantas daninhas.

Table 6. Percentage of leaf temperature in relation to the control (TF\%) of eucalyptus plants in different periods of cohabitation with weeds.

\begin{tabular}{|c|c|c|c|}
\hline \multirow[t]{2}{*}{ Períodos de convivência } & \multicolumn{3}{|c|}{ Espécies } \\
\hline & Commelinea bengalensis & Ipomoeia grandifolia & Urochloa brizantha \\
\hline Testemunha & $100,00 *$ & 100,00 & 100,00 \\
\hline 60 dias & 98,49 & 100,90 & 99,21 \\
\hline 105 dias & 96,83 & 100,89 & 100,98 \\
\hline $\mathrm{CV}(\%)$ & & 1,25 & \\
\hline
\end{tabular}

*Não significativo.

*Not significant.

Ao se avaliar a porcentagem da relação carbono interno/carbono atmosférico $(\mathrm{Ci} / \mathrm{Ca} \%)$ em plantas de eucalipto, constatou-se que a cultura mostrou maiores valores referentes a esta variável quando competindo com a trapoeraba em relação às demais espécies avaliadas aos 60 e 105 dias de convivência. Ao se avaliar o efeito do período de convivência dentro de cada espécie, constataram-se maiores valores de $\mathrm{Ci} / \mathrm{Ca}$ nas plantas de eucalipto aos 60 e 105 dias diferindo da testemunha cultivada isoladamente para todas as espécies avaliadas (Tabela 7). 
FAUSTINO, L.A. et al. Eucalipto em convivência com plantas daninhas.

Tabela 7. Porcentagem da relação carbono interno/carbono atmosférico em relação à testemunha $(\mathrm{Ci} / \mathrm{Ca} \%)$ de plantas de eucalipto em diferentes períodos de convivência com plantas daninhas.

Table 7. Percentage of the internal carbon/atmospheric carbon ratio in relation to the control $(\mathrm{Ci} / \mathrm{Ca} \%)$ of eucalyptus plants in different periods of cohabitation with weeds.

\begin{tabular}{lccc}
\hline Períodos de convivência & \multicolumn{3}{c}{ Espécies } \\
\cline { 2 - 4 } & Commelinea bengalensis & Ipomoeia grandifolia & Urochloa brizantha \\
\hline Testemunha & $100,00 \mathrm{~B}^{*}$ & $100,00 \mathrm{~B}$ & $100,00 \mathrm{~B}$ \\
60 dias & $182,05 \mathrm{a} \mathrm{A}$ & $126,53 \mathrm{~b} \mathrm{~A}$ & $134,29 \mathrm{~b} \mathrm{~A}$ \\
105 dias & $181,54 \mathrm{a} \mathrm{A}$ & $131,37 \mathrm{~b} \mathrm{~A}$ & $139,76 \mathrm{~b} \mathrm{~A}$ \\
\hline CV $(\%)$ & & 4,44 & \\
\hline
\end{tabular}

*Médias seguidas pela mesma letra minúscula na linha e maiúscula na coluna não diferem entre si pelo Teste de Tukey a $5 \%$ de probabilidade de erro.

*Averages followed by the same lowercase letter in the row and uppercase in the column do not differ from each other by the Tukey test at $5 \%$ probability of error.

O fechamento dos estômatos normalmente reduz a entrada de $\mathrm{CO}_{2}$ na câmara subestomática, diminuindo a quantidade de substrato disponível para o ciclo de Calvin. Entretanto, ao se avaliar a $\mathrm{Ci} / \mathrm{Ca} \%$, que é uma relação direta entre a concentração de $\mathrm{CO}_{2}$ na câmara subestomática e a concentração de $\mathrm{CO}_{2}$ no ambiente, observa-se que o maior valor da $\mathrm{Ci} / \mathrm{Ca}$ encontrado, quando o eucalipto esteve em competição com cada uma das espécies daninhas avaliadas, indica que o eucalipto não está consumindo $\mathrm{o} \quad \mathrm{CO}_{2}$ normalmente e que a redução na fotossíntese está ocorrendo provavelmente em função da redução dos teores de nutrientes no solo causado pela competição entre as plantas daninhas e a cultura. $\mathrm{O}$ nitrogênio, o fósforo, o enxofre juntamente com os micronutrientes ferro, manganês e cobre estão presentes em diversas proteínas relacionadas ao transporte de elétrons nas membranas dos tilacoídes do cloroplasto, assim a deficiência de qualquer um destes nutrientes proporciona efeito direto na fotossíntese (Taiz e Zeiger, 2013).

A porcentagem da eficiência do uso da água em plantas de eucalipto em relação à testemunha (EU\%), não diferiu entre as espécies cultivadas em convivência com a cultura.
Entretanto, ao se avaliar o efeito do período de convivência dentro das três espécies, constatou-se redução da EU\% em plantas de eucalipto aos 60 e 105 dias em relação às parcelas cultivadas na ausência de competição para todas as espécies avaliadas (Tabela 8).

A eficiência do uso de água é caracterizada como a quantidade de água evapotranspirada por uma planta para a produção de determinada quantidade de matéria seca. Desse modo, plantas mais eficientes no uso de água acumulam mais matéria seca por grama de água transpirada (Baptista et al., 2012). O uso mais eficiente de água está diretamente associado ao tempo de abertura estomática, pois, enquanto a planta absorve $\mathrm{CO}_{2}$ para a fotossíntese, a água é perdida para o ambiente por evapotranspiração, seguindo uma corrente de potenciais hídricos (Pereira Netto, 2002). No presente trabalho, a eficiência no uso da água mostrou redução com o incremento do período de convivência da cultura com as três espécies daninhas estudadas (Tabela 8), desse modo, o aumento do período de competição promoveu redução da eficiência no uso da água das plantas de eucalipto, principalmente devido à redução da taxa fotossintética que está diretamente relacionada ao uso da água e taxa transpiratória. 
FAUSTINO, L.A. et al. Eucalipto em convivência com plantas daninhas.

Tabela 8. Porcentagem da eficiência do uso da água em relação à testemunha (EU\%) de plantas de eucalipto em diferentes períodos de convivência com plantas daninhas.

Table 8. Percentage of water use efficiency in relation to the control (US\%) of eucalyptus plants in different periods of weed coexistence.

\begin{tabular}{lccc}
\hline Períodos de convivência & \multicolumn{3}{c}{ Espécies } \\
\cline { 2 - 4 } & Commelinea bengalensis & Ipomoeia grandifolia & Urochloa brizantha \\
\hline Testemunha & $100,00 \mathrm{~A}^{*}$ & $100,00 \mathrm{~A}$ & $100,00 \mathrm{~A}$ \\
60 dias & 36,39 a B & 49,01 a B & 42,01 a B \\
105 dias & 27,80 a B & 41,08 a B & 38,69 a B \\
\hline CV $(\%)$ & & 17,96 & \\
\hline
\end{tabular}

*Médias seguidas pela mesma letra minúscula na linha e maiúscula na coluna não diferem entre si pelo Teste de Tukey a $5 \%$ de probabilidade de erro.

*Averages followed by the same lowercase letter in the row and uppercase in the column do not differ from each other by the Tukey test at $5 \%$ probability of error.

\section{CONCLUSÕES}

De acordo com os resultados, conclui-se que as três espécies avaliadas promoveram efeitos negativos nas características fisiológicas das plantas de eucalipto, promovendo redução na taxa fotossintética e na eficiência do uso da água, além de incremento na porcentagem, na concentração do carbono interno e na taxa transpiratória. As três espécies avaliadas afetaram de maneira similar as características fisiológicas do da cultura.

\section{AGRADECIMENTOS}

Os autores agradecem à Coordenação de Aperfeiçoamento de Pessoal de Nível Superior CAPES e Fundação de Amparo à Pesquisa do Estado de Minas Gerais - FAPEMIG pelo apoio financeiro e as bolsas concedidas.

\section{REFERÊNCIAS BIBLIOGRÁFICAS}

BAPTISTA, J.M.; ALMEIDA, M.D.; VIEIRA, P. Programa nacional para o uso eficiente da água. Instituto Superior de Agronomia, v. 1, n. 1, 2012. Disponível em: $<$ https://www.apambiente.pt/_zdata/ CONSULTA_PUBLICA/2012/ PNUEA/ Implementacao-PNUEA_2012-2020_JUNHO.pdf>. Acesso em: 17 fev. 2017.

CONCENÇO, G. et al. Uso da água em biótipos de azevém (Lolium multiflorum) em condição de competição. Planta Daninha, v. 25, n. 3, p. 449-455, 2007.

. et al. Uso da água por plantas de arroz em competição com biótipos de Echinochloa crusgalli resistente e suscetível ao herbicida quinclorac. Planta Daninha, v. 27, n. 2, p. 249-256, 2009.

FAUSTINO, L.A. Convivência de eucalipto com Commelina benghalensis. 2015. 40 f. Dissertação (Mestrado em Fitotecnia) - Universidade Federal de Viçosa, Viçosa, MG. 
FERREIRA, G.L. et al. Eucalypt growth submitted to management of Urochloa spp. Planta Daninha, v. 34, n. 1, p. 99-107, 2016.

FLOSS, E.L. Fisiologia das plantas cultivadas. Passo Fundo: Universidade de Passo Fundo, 2011. v. 4,734 p.

GALON, L. et al. Estimativa das perdas de produtividade de grãos em cultivares de arroz (Oryza sativa) pela interferência do capim-arroz (Echinochloa spp.). Planta Daninha, v. 25 , n. 3, p. 697-707, 2007.

JONES, H.G. Stomatal control of photosynthesis and transpiration. Journal of Experimental Botany, v. 49, p. 387-398, 1998. Special Issue.

MACHADO, A.F.L. et al. Eficiência fotossintética e uso da água em plantas de eucalipto pulverizadas com glyphosate. Planta Daninha, v. 28, n. 2, p. 319-327, 2010.

MATOS, C.C. et al. Características fisiológicas do cafeeiro em competição com plantas daninhas. Bioscience Journal, v. 29, n. 5, p. 1111-1119, 2013.

MELO, P.T.B.S. et al. Comportamento de populações de arroz irrigado em função das proporções de plantas originadas de sementes de alta e baixa qualidade fisiológica. Revista Brasileira de Agrociência, v. 12, n. 1, p. 37-43, 2006.

MESSINGER, S.M. et al. Evidence for involvement of photosynthetic processes in the stomatal response to $\mathrm{CO}_{2}$. Plant Physiology, v. 140, n. 2, p. 771-778, 2006.
PEREIRA-NETTO, A.B. Crescimento e desenvolvimento. In: WACHOWICZ, C.M.; CARVALHO, R.I.N. (Ed.). Fisiologia vegetal produção e pós-colheita. Curitiba: Champagnat, 2002. p. 17-42.

RADOSEVICH, S.; HOLT, J.; GHERSA, C. Physiological aspects of competition. In: STEVEN, R. et al. (Ed.). Weed ecology implicatios for managements. New York: John Willey and Sons, 1996. p. 217-301.

RIGOLI, R.P. et al. Habilidade competitiva relativa do trigo (Triticum aestivum) em convivência com azevém (Lolium multiflorum) ou nabo (Raphanus raphanistrum). Planta Daninha, v. 26, n. 1, p. 93-100, 2008.

SHARKEY, T.D.; RASCHKE, K. Effect of light quality on stomatal opening in leaves of Xanthium strumarium L. Plant Physiology, v. 68, n. 5 , p. 1170-1174, 1981.

SILVA, A.A. et al. Herbicidas: classificação e mecanismo de ação. In: SILVA, A.A.; SILVA, J.F. (Ed.). Tópicos em manejo de plantas daninhas. Viçosa: Universidade Federal de Viçosa, 2007. p. 83-148.

SILVA, W. et al. Taxa fotossintética líquida de Eucalyptus citriodora Hook e E. grandis W. Hill em resposta à diferentes níveis de água no solo e associação com Brachiaria brizantha Stapf. Acta Scientiarum, v. 23, n. 5, p. 1205-1209, 2001.

TAIZ, L.; ZEIGER, E. Fisiologia vegetal. Porto Alegre: Artmed, 2013. v. 5, 954 p. 
FAUSTINO, L.A. et al. Eucalipto em convivência com plantas daninhas.

TYREE, M.T.; SPERRY, J.S. Do woody plants operate near the point of catastrophic xylem dysfunction caused by dynamic water stress? Answers from a model. Plant Physiology, v. 88, n. 3, p. 574-580, 1988.

VIDAL, R.A. et al. Nível de dano econômico de

Brachiaria plantaginea na cultura de milho irrigado. Planta Daninha, v. 22, n. 1, p. 63-69, 2004.

WINKLER, L.M.; VIDAL, R.A.; BARBOSA NETO, J.F. Aspectos genéticos envolvidos na resistência de plantas daninhas aos herbicidas. Plantio Direto, v. 70, n. 1, p. 21-24, 2002. 